\title{
A repeated measures study of phenol, paraben and Triclocarban urinary biomarkers and circulating maternal hormones during gestation in the Puerto Rico PROTECT cohort
}

\author{
Amira M. Aker ${ }^{1}$, Kelly K. Ferguson ${ }^{1,2}$, Zaira Y. Rosario ${ }^{3}$, Bhramar Mukherjee ${ }^{4}$, Akram N. Alshawabkeh ${ }^{5}$
} Antonia M. Calafat ${ }^{6}$, José F. Cordero ${ }^{7}$ and John D. Meeker ${ }^{1^{*}}$

\begin{abstract}
Introduction: Prenatal exposure to some phenols and parabens has been associated with adverse birth outcomes. Hormones may play an intermediate role between phenols and adverse outcomes. We examined the associations of phenol and paraben exposures with maternal reproductive and thyroid hormones in 602 pregnant women in Puerto Rico. Urinary triclocarban, phenol and paraben biomarkers, and serum hormones (estriol, progesterone, testosterone, sex-hormone-binding globulin (SHBG), corticotropin-releasing hormone (CRH), total triiodothyronine (T3), total thyroxine (T4), free thyroxine (FT4) and thyroid-stimulating hormone (TSH)) were measured at two visits during pregnancy.

Methods: Linear mixed models with a random intercept were constructed to examine the associations between hormones and urinary biomarkers. Results were additionally stratified by study visit. Results were transformed to hormone percent changes for an inter-quartile-range difference in exposure biomarker concentrations (\% $\Delta$ ).

Results: Bisphenol-S was associated with a decrease in CRH [(\% $\Delta-11.35 ; 95 \% \mathrm{Cl}:-18.71,-3.33)$, and bisphenol-F was associated with an increase in FT4 (\% $\Delta: 2.76 ; 95 \%$ Cl: $0.29,5.22)$. Butyl-, methyl- and propylparaben were associated with decreases in SHBG [(\% $\Delta:-5.27 ; 95 \% \mathrm{Cl}:-9.4,-1.14)$; (\% $\Delta:-3.53 ; 95 \% \mathrm{Cl}:-7.37,0.31) ;(\% \Delta:-3.74 ; 95 \% \mathrm{Cl}:-7.76$, 0.27)]. Triclocarban was positively associated with T3 (\% $\Delta: 4.08 ; 95 \% \mathrm{Cl}: 1.18,6.98)$ and T3/T4 ratio $(\% \Delta: 4.67 ; 95 \% \mathrm{Cl}:-1.37$, 6.65), and suggestively negatively associated with TSH (\% $\Delta:-10.12 ; 95 \% \mathrm{Cl}:-19.47,0.32)$. There was evidence of susceptible windows of vulnerability for some associations. At 24-28 weeks gestation, there was a positive association between 2,4-dichlorophenol and $\mathrm{CRH}(\% \Delta: 9.66 ; 95 \% \mathrm{Cl}: 0.67,19.45)$ and between triclosan and estriol (\% 1 : 13.17; $95 \% \mathrm{Cl}: 2.34,25.2)$; and a negative association between triclocarban and SHBG (\% 1 : -9.71 ; 95\% Cl:-19.1, - 0.27) and between bisphenol $\mathrm{A}$ and testosterone (\% $\Delta$ : $-17.37 ; 95 \% \mathrm{Cl}:-26.7,-6.87)$.
\end{abstract}

Conclusion: Phenols and parabens are associated with hormone levels during pregnancy. Further studies are required to substantiate these findings.

Keywords: Thyroid hormones, Reproductive hormones, Pregnancy, In-utero, Endocrine disruption, Phenols, Parabens, Triclocarban

\footnotetext{
* Correspondence: meekerj@umich.edu

${ }^{1}$ Department of Environmental Health Sciences, University of Michigan

School of Public Health, Room 1835 SPH I, 1415 Washington Heights, Ann

Arbor, MI 48109-2029, USA

Full list of author information is available at the end of the article
}

(c) The Author(s). 2019 Open Access This article is distributed under the terms of the Creative Commons Attribution 4.0 International License (http://creativecommons.org/licenses/by/4.0/), which permits unrestricted use, distribution, and reproduction in any medium, provided you give appropriate credit to the original author(s) and the source, provide a link to the Creative Commons license, and indicate if changes were made. The Creative Commons Public Domain Dedication waiver (http://creativecommons.org/publicdomain/zero/1.0/) applies to the data made available in this article, unless otherwise stated. 


\section{Background}

Exposure to phenols and parabens has been linked to various adverse health effects, including ovarian toxicity, cancer, and adverse neurodevelopmental outcomes [1-4]. Prenatal exposure to these chemicals, in particular, may have a long lasting effect on fetal health into adulthood. For example, prenatal exposure to phenols and parabens has been linked to adverse birth outcomes $[5,6]$, respiratory health effects in children [7], and cardiometabolic risk [8]. The exact mechanisms at play are still not fully understood; however, endocrine disruption is hypothesized to be one of the main toxicity pathways [3, 9-11].

Reproductive and thyroid hormones play an essential role in the maintenance of pregnancy and the development of the fetus [12-16], therefore pregnancy is a vulnerable window for endocrine disruption due to the varying levels of hormones involved in the growing organism [17]. Endocrine disrupting chemicals could act through several pathways, including hormone synthesis, regulation, transport and metabolism, and/or interference with receptors. Phenols and parabens have estrogenic and androgenic properties [1, 18-20], but few human studies have looked into the effect of these chemicals on maternal hormones during pregnancy. Most existing studies in this area use smaller study populations or only examined a single time point in pregnancy, which do not capture the changing hormone levels and high variability of phenols and paraben exposure during pregnancy. Furthermore, no or few studies explored the associations between these chemicals and maternal testosterone, corticotropin-releasing hormone (CRH), sex hormone-binding globulin (SHBG) and estriol, all of which play essential roles in maintaining healthy pregnancies.

Given the growing evidence of the endocrine disrupting effects of phenols and parabens [18, 21-25], our aim was to study the relationships between phenols and parabens on reproductive and thyroid hormones in our ongoing cohort of pregnant women in Puerto Rico. The study follows the women over multiple time points during pregnancy, providing more power than previous studies, and allows for the identification of potential windows of susceptibility. We previously reported early preliminary results on associations between select phenols and parabens with hormones in this Puerto Rican cohort [26]. This manuscript is an update of our previous results that utilizes a much larger sample size, includes additional hormones (estriol, testosterone, total triiodothyronine, and total thyroxine), as well as additional exposure biomarkers yet to be studied in detail (ethylparaben, BPS, BPF and triclocarban). Due to the lack of human health data, this study was exploratory in nature, with the exception of $\mathrm{BPA}$, triclosan, methylparaben and propylparaben. We hypothesized a decrease in serum thyroid hormone levels in association with triclosan, methyl- and propyl-paraben, and an increase in serum thyroid hormones with BPA concentrations.

\section{Methods \\ Study participants}

Participants for the present study were from an ongoing prospective cohort of pregnant women in Puerto Rico, named the Puerto Rico Testsite for Exploring Contamination Threats (PROTECT) cohort. Details on the recruitment and inclusion criteria have been described previously [27, 28]. The study participants included in the present analysis were recruited from 2012 to 2017 at $14 \pm 2$ weeks gestation from two hospitals and five affiliated prenatal clinics in Northern Puerto Rico. They were aged between 18 and 40 years. The exclusion criteria included women who lived outside the region, had multiple gestations, used oral contraceptives within 3 months prior to getting pregnant, got pregnant using in vitro fertilization, or had known medical health conditions (diabetes, hypertension, etc.). Three visits were conducted with the study participants to coincide with periods of rapid fetal growth and routine clinical visits (Visit 1: 16-20; Visit 2: 20-24; Visit 3: 24-28 gestation weeks). Demographic information was collected via questionnaires at the initial study visit. Spot urine samples were collected at the three study visits, whereas blood samples were collected during the first and third visits.

The present analysis includes 602 women recruited into the study (of the total 1311 women enrolled in the cohort to date) for whom both total phenol and paraben concentrations and hormone measurements from at least one study visit were available. This study was approved by the research and ethics committees of the University Of Michigan School Of Public Health, University of Puerto Rico, Northeastern University, and the University of Georgia. All study participants provided full informed consent prior to participation. The involvement of the Centers for Disease Control and Prevention (CDC) laboratory did not constitute engagement in human subjects research.

\section{Quantification of urinary biomarkers}

After collection, spot urine samples were divided into aliquots and frozen at $-80^{\circ} \mathrm{C}$ until they were shipped overnight with dry ice to the $\mathrm{CDC}$ for analysis. Urine samples were analyzed for seven phenols (2,4-dichlorophenol, 2,5-dichlorophenol, BPA, BPS, BPF, benzophenone-3, triclosan), triclocarban, and four parabens (ethylparaben, methylparaben, butylparaben, propylparaben) using online solid phase extraction-high-performance liquid chromatographyisotope dilution tandem mass spectrometry [29-31]. Biomarker concentrations below the limit of detection (LOD) were assigned a value of the LOD divided by $\sqrt{2}$ [32]. The LODs were as follows: $0.1 \mu \mathrm{g} / \mathrm{L}$ (2,4-dichlorphenol, 
2,5-dichlorophenol, BPS, triclocarban, butylparaben, propylparaben); $0.2 \mu \mathrm{g} / \mathrm{L}$ (BPA, BPF); $0.4 \mu \mathrm{g} / \mathrm{L}$ (benzophenone-3); $1 \mu \mathrm{g} / \mathrm{L}$ (methylparaben, ethylparaben); and $1.7 \mu \mathrm{g} / \mathrm{L}$ (triclosan). Urinary dilution was accounted for by using urinary specific gravity (SG), and was measured using a digital handheld refractometer (AtagoCo., Ltd., Tokyo, Japan). For preliminary data analysis, urinary biomarker concentrations were corrected for SG using the following formula:

$$
\mathrm{P}_{\mathrm{C}}=\mathrm{M}\left[\left(\mathrm{SG}_{\mathrm{m}}-1\right) /\left(\mathrm{SG}_{\mathrm{i}}-1\right)\right]
$$

where $P_{c}$ is the SG-corrected concentration $(\mu \mathrm{g} / \mathrm{L}), \mathrm{M}$ is the measured concentration, $\mathrm{SG}_{\mathrm{m}}$ is the study population median urinary specific gravity (1.0196), and $S G_{i}$ is the individual's urinary specific gravity. The sample size for BPF, BPS, triclocarban and ethylparaben was smaller than the rest of the biomarkers because they were only quantified in a later sub-sample of the cohort.

\section{Hormone measurement}

Serum samples were collected during visits 1 and 3 . Volume limitations resulted in differences in the number of samples analyzed by hormone. All hormone analyses were conducted at the Central Ligand Assay Satellite Services (CLASS) laboratory, Department of Epidemiology, School of Public Health, University of Michigan. Progesterone, SHBG, testosterone, total triiodothyronine (T3), total thyroxine (T4), free thyroxine (FT4), and thyroid-stimulating hormone (TSH) were measured in serum using a chemiluminescence immunoassay (ADVIA Centaur ${ }^{\circ}$ CP Immunoassay System, Seimens Healthineers). Estriol and CRH were measured in serum using an enzyme immunoassay (Estriol ELISA Kit, ALPCO; CRH ELISA Kit, LifeSpan BioSciences). In addition to measured hormones, the ratio of progesterone to estriol (Prog/Estriol Ratio), and the ratio of $\mathrm{T} 3$ and $\mathrm{T} 4$ (T3/T4 ratio) were calculated for the purposes of this analysis. Hormone ratios may be a better indicator of adverse pregnancy outcomes (such as preterm birth) than the individual hormones alone [33-35]. Two samples had a TSH level below the LOD. Because this result was not biologically plausible, these two values were dropped from the analyses.

\section{Statistical analyses}

Distributions of key demographic characteristics were calculated. All urinary exposure biomarkers, and the serum hormones progesterone, estriol, $\mathrm{CRH}$, TSH and progesterone/estriol ratio were positively-skewed, and were natural log-transformed. The distributions of SHBG, FT4, T3, T4 and T3/T4 ratio approximated normality and remained untransformed in all analyses. Geometric means and standard deviations were calculated for all SG-corrected exposure biomarkers, hormones, and the ratios of progesterone/estriol and T3/T4. We examined urinary exposure biomarkers concentrations and serum hormone levels by study visit, and calculated Spearman correlations between unlogged average SG-corrected exposure biomarkers. To assess differences in exposure biomarkers and hormones across study visits, we ran Linear Mixed Models (LMM) with a subject-specific random intercept regressing the biomarker or hormone against the study visit. Specific gravity was used as a covariate in the model instead of using the SG-corrected biomarker concentrations. The selection of a random intercept and slope was determined using BIC values. BPF and ethylparaben were detected in less than $50 \%$ of the samples. Therefore, we transformed BPF and ethylparaben into dichotomous variables, where 0 represented concentrations below the LOD, and 1 represented detectable concentrations. These categorical BPF and ethylparaben variables were used in all of the following regression analyses.

In our repeated measures analysis, we regressed one hormone or hormone ratio on one urinary biomarker using LMM, with a subject-specific random intercept for each model to account for intra-individual correlation of serial hormone measurements collected over the two study visits. The urinary biomarker concentrations at the two visits were treated as time-varying variables in the LMM models. Crude models included specific gravity and study visit as covariates. Potential confounders were selected a priori from the existing literature, and included as covariates if they were found to change the main effect estimate by $>10 \%$. Final models were adjusted for specific gravity, study visit, body mass index (BMI) at the first study visit, maternal age, the number of hours of second-hand smoking exposure per day, and a socio-economic variable. All covariates, except for maternal age and specific gravity, were categorical. The socio-economic variable used in the model differed by the hormone regressed. Maternal education was a strong confounder for models regressing progesterone, estriol, and progesterone/estriol ratio against urinary biomarkers concentrations, and was used as the socio-economic index for those models. All other models used insurance type as the socio-economic status index. The selection of the socio-economic variable was based on the percent change in the main effect estimate, and the $p$ value of the socio-economic variable in final models.

To assess windows of vulnerability, we ran two more analyses. First, we ran the same LMMs regressing hormones and urinary biomarkers concentrations with an interaction term between the urinary biomarker and the study visit. Second, we ran multiple linear regressions (MLR) stratified by study visit of sample collection. The MLR models were adjusted for the same covariates as those in the LMMs. 
To increase interpretability of our results, we transformed regression coefficients to percent changes (and associated 95\% confidence intervals, CIs) in hormone concentration in relation to the interquartile range (IQR) increase in urinary biomarker concentrations. Beta coefficients from models with categorical biomarkers (BPF and ethylparaben) were transformed to percent changes (and associated 95\% confidence intervals) in hormone concentration at detectable vs non-detectable biomarker concentrations. The alpha level was set at 0.05 . All statistical analyses were conducted in R Version 3.4.2.

As a sensitivity analysis, all models were re-run using specific gravity as a covariate in combination with exposure biomarkers corrected for specific gravity as was described by O'Brien et al. [36]. We observed no differences in our results, and therefore, retained our original models using un-corrected exposure biomarkers with specific gravity included as a covariate.

\section{Results}

The 602 study participants had a mean age of 26.4 and approximately $60 \%$ had BMI levels below $30 \mathrm{~kg} / \mathrm{m}^{2}$ (Table 1). Although the majority of women reported never smoking (75\%), 4\% reported currently smoking, and $7 \%$ reported exposure to second-hand smoking for more than an hour per day. Six percent reported consuming alcohol in the last few months. A quarter of the study participants reported a household income of less than $\$ 10,000$, and only $11 \%$ reported a household income $>\$ 50,000$. A quarter of the participants did not report their incomes. As compared to the overall PROTECT cohort, the study participants included in the present analysis had higher rates of smoking, and had overall lower household income and education levels.

The exposure biomarkers included in this analysis were highly detected in the study population, with the exception of ethylparaben and BPF (Table 2). BPF was detected in between 50 and $60 \%$ of the study sample; ethylparaben was detected in between 42 and $54 \%$ of the sample, depending on study visit. Concentrations of urinary biomarkers remained relatively consistent across the two study visits, with the exception of a decrease in BPA $(p<0.001)$ and butylparaben $(p=0.04)$. There was an increase in most hormones across the two study visits, particularly progesterone, estriol, SHBG and CRH. T4 levels remained consistent from 16 to 20 and 24-28 weeks gestation.

Methylparaben and propylparaben were strongly correlated [Spearman correlation of $0.8(p<0.001)$ ] (Fig. 1). Ethylparaben and butylparaben showed moderate correlation with methylparaben and propylparaben with Spearman correlations between $0.33-0.47$ ( $p$ values $<0.001$ ). 2,4-Dichlorophenol and 2,5-dichlorophenol showed a fairly strong correlation (Spearman $r=0.6, p<0.001$ ). Triclosan
Table 1 Summary demographics and differences between the PROTECT study participants included in present analysis versus participants not included because of missing urine and/or serum samples

\begin{tabular}{lcll}
\hline Total N & Included & Not Included & $p$ \\
& 602 & 709 & \\
\hline Age (mean [SD]) & $26.51(5.66)$ & $26.94(5.34)$ & 0.25 \\
& BMl in kg/m $(\%)$ & & \\
$<25$ & $245(40.7)$ & $192(27.1)$ & 0.99 \\
$25-30$ & $114(18.9)$ & $87(12.3)$ & \\
$>30$ & $73(12.1)$ & $56(7.9)$ & \\
Missing & $170(28.2)$ & $374(52.8)$ & \\
& & & \\
Never & Current Smoker (\%) & & \\
Ever & $440(73.1)$ & $323(45.6)$ & 0.03 \\
Current & $63(10.5)$ & $57(8.0)$ & \\
Missing & $23(3.8)$ & $6(0.8)$ & \\
& $76(12.6)$ & $323(45.6)$ &
\end{tabular}

Exposure to Second-Hand Smoking per Day (\%)

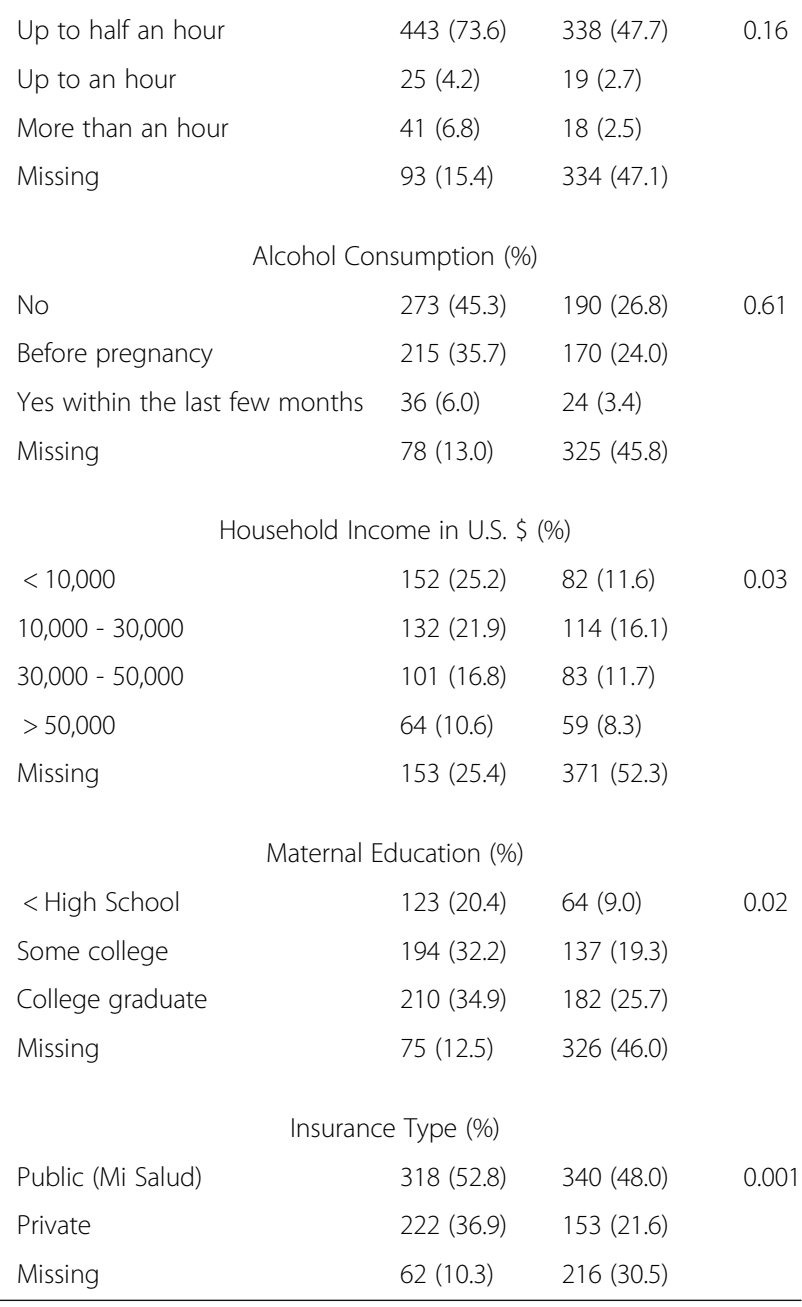


Table 2 Distribution of SG-corrected urinary biomarker concentrations and hormones and differences by study visit of sample collection in pregnancy

\begin{tabular}{|c|c|c|c|c|c|c|c|c|c|c|c|c|c|}
\hline \multirow[t]{2}{*}{ Biomarkers $^{a}$} & \multicolumn{6}{|c|}{$16-20$ weeks $(N=389)$} & \multicolumn{6}{|c|}{ 24-28 weeks $(N=262)$} & \multirow[t]{2}{*}{$p$-value } \\
\hline & GM (GSD) & $\%<\mathrm{LOD}$ & $25 \%$ & $50 \%$ & $75 \%$ & $95 \%$ & GM (GSD) & $\%<\mathrm{LOD}$ & $25 \%$ & $50 \%$ & $75 \%$ & $95 \%$ & \\
\hline 2,4-DCP & $1.17(3.24)$ & 0.5 & 0.52 & 0.93 & 2.0 & 10.7 & $1.13(9.8)$ & 2.3 & 0.46 & 0.86 & 2.19 & 12.9 & 0.65 \\
\hline 2,5-DCP & $14.03(5.14)$ & 0.3 & 4.57 & 10.4 & 30.2 & 432.6 & $13.63(360.3)$ & 0 & 4.63 & 9.61 & 26.53 & 429.6 & 0.70 \\
\hline BPA & $2.31(2.25)$ & 0.3 & 1.33 & 2.14 & 3.36 & 9.56 & $1.88(2.4)$ & 0.8 & 1.14 & 1.83 & 3.0 & 6.18 & $<0.001^{*}$ \\
\hline BPS $^{\mathrm{i}}$ & $0.54(3.15)$ & 3.4 & 0.23 & 0.50 & 1.07 & 4.01 & $0.54(5.2)$ & 8.6 & 0.23 & 0.47 & 1.06 & 4.23 & 0.95 \\
\hline $\mathrm{BPF}^{\mathrm{i}}$ & $0.35(3.18)$ & 51.9 & $<\mathrm{LOD}$ & 0.25 & 0.56 & 2.88 & $0.31(2.4)$ & 59.9 & $<\mathrm{LOD}$ & 0.24 & 0.46 & 2.09 & 0.22 \\
\hline $\mathrm{BP}-3$ & $38.34(6.49)$ & 0.5 & 10.6 & 22.4 & 110.8 & 1547 & $44.27(2500.4)$ & 0.8 & 11.9 & 25.3 & 160.7 & 1913.5 & 0.69 \\
\hline TCS & $21.78(8.72)$ & 11.1 & 3.70 & 13.1 & 146.8 & 877.4 & $25.03(327.4)$ & 6.1 & 4.73 & 17.9 & 118.6 & 960.2 & 0.64 \\
\hline $\mathrm{TCC}^{\mathrm{i}}$ & $4.34(10.27)$ & 5.8 & 0.70 & 3.36 & 33.6 & 157.7 & $4.86(56.2)$ & 5.6 & 0.78 & 4.78 & 32.76 & 168.6 & 0.46 \\
\hline$E B^{i}$ & $3.42(7.73)$ & 42.4 & $<\mathrm{LOD}$ & 1.66 & 15.4 & 177.5 & $2.55(62.1)$ & 54 & $<\mathrm{LOD}$ & $<\mathrm{LOD}$ & 7.6 & 76.1 & 0.12 \\
\hline MPB & $80.72(5.06)$ & 0.3 & 25.08 & 116.5 & 274.8 & 846.1 & $92.5(359)$ & 0.8 & 30.2 & 111 & 314.5 & 1054.9 & 0.18 \\
\hline BPB & $0.55(8.12)$ & 23.8 & 0.10 & 0.25 & 2.17 & 39.1 & $0.42(12.2)$ & 33.5 & 0.10 & 0.2 & 0.91 & 32.6 & $0.04^{*}$ \\
\hline PPB & $17.51(7.19)$ & 0 & 3.59 & 21.1 & 80.5 & 262.4 & $17.61(111.4)$ & 0.4 & 4.0 & 25.17 & 83.65 & 253.8 & 0.99 \\
\hline \multirow[t]{2}{*}{ Hormones } & \multicolumn{6}{|c|}{$16-20$ weeks $(N=483)$} & \multicolumn{6}{|c|}{ 24-28 weeks $(\mathrm{N}=389)$} & \multirow[t]{2}{*}{$p$-value } \\
\hline & GM (GSD) & $\%<\mathrm{LOD}$ & $25 \%$ & $50 \%$ & $75 \%$ & $95 \%$ & GM (GSD) & $\%<\mathrm{LOD}$ & $25 \%$ & $50 \%$ & $75 \%$ & $95 \%$ & \\
\hline Progesterone $^{b}$ & $49.63(1.49)$ & 0 & 37.2 & 48.5 & 61.6 & 98.2 & $95.94(1.65)$ & 0 & 70.9 & 90.9 & 129.2 & 222.7 & $<0.001^{*}$ \\
\hline Estriol $^{b}$ & $18.85(1.76)$ & 0 & 13.2 & 17.6 & 27.5 & 50.5 & $44.63(1.59)$ & 0 & 33.8 & 44.9 & 57.5 & 97.3 & $<0.001^{*}$ \\
\hline SHBG ${ }^{c}$ & $575.22(1.4)$ & 0 & 482.4 & 588.6 & 703.8 & 907.9 & $670.19(1.35)$ & 0 & 536.3 & 672.9 & 832.6 & 1097.7 & $<0.001^{*}$ \\
\hline Prog/Estriol & 2.64 (1.69) & - & 1.97 & 2.66 & 3.70 & 5.83 & $2.15(1.62)$ & - & 1.58 & 2.12 & 2.86 & 4.48 & $<0.001^{*}$ \\
\hline $\mathrm{CRH}^{\mathrm{d}}$ & 76.67 (1.70) & 0 & 54.8 & 80.6 & 111.8 & 171.9 & 78.01 (1.76) & 0 & 55.2 & 82.8 & 114.3 & 178.1 & $<0.001^{*}$ \\
\hline Testosterone $^{f}$ & $50.55(1.81)$ & 1.8 & 37.5 & 51.5 & 73.5 & 124.4 & $60.22(1.75)$ & 0.9 & 45.1 & 60.7 & 88.1 & 131.6 & $<0.001^{*}$ \\
\hline $\mathrm{TSH}^{\mathrm{e}}$ & $1.29(2.14)$ & 0 & 0.93 & 1.38 & 2.06 & 3.28 & 1.45 (1.79) & 0 & 1.08 & 1.51 & 2.05 & 3.64 & $0.04^{*}$ \\
\hline $\mathrm{FT}_{4}^{\mathrm{f}}$ & $1.10(1.13)$ & 0 & 1.02 & 1.10 & 1.19 & 1.35 & $1.06(1.13)$ & 0 & 0.98 & 1.06 & 1.15 & 1.29 & $<0.001^{*}$ \\
\hline $\mathrm{T} 3^{\mathrm{b}}$ & $1.94(1.22)$ & 0 & 1.71 & 1.97 & 2.22 & 2.59 & $1.95(1.21)$ & 0 & 1.72 & 1.99 & 2.24 & 2.67 & $0.03^{*}$ \\
\hline $\mathrm{T} 4^{\mathrm{g}}$ & $11.90(1.20)$ & 0 & 10.7 & 11.95 & 13.3 & 15.7 & $11.71(1.20)$ & 0 & 10.5 & 11.7 & 13.2 & 15.5 & 0.27 \\
\hline $\mathrm{T} 3 / \mathrm{T} 4$ & $0.16(1.21)$ & - & 0.14 & 0.16 & 0.19 & 0.23 & $0.17(1.22)$ & - & 0.15 & 0.17 & 0.19 & 0.23 & $<0.001^{*}$ \\
\hline
\end{tabular}

GM Geometric mean, GSD Geometric standard deviation

2,4-DCP: 2,4-dichlorophenol; 2,5-DCP: 2,5-dichlorophenol; BP-3 Benzophenone, TCS Triclosan, TCC Triclocarban, EPB ethylparaben, MPB Methylparaben, BPB Butylparaben, PPB Propylparaben

Range of gestational weeks: 16-20 weeks: 16-20 weeks gestation, 24-28 weeks: 24-28 weeks gestation

"Significant difference $(p<0.05)$ in urinary biomarker or hormone compared to reference $(16-20$ weeks) using linear mixed models with a random intercept

anits: $\mu \mathrm{g} / \mathrm{L}$. ${ }^{\mathrm{b}}$ Units: $\mathrm{ng} / \mathrm{mL}$. ${ }^{\mathrm{a}}$ Units: $\mathrm{nmol} / \mathrm{L} .{ }^{\mathrm{d}}$ Units: $\mathrm{pg} / \mathrm{mL} .{ }^{\mathrm{e}}$ Units: $\mathrm{ulU} / \mathrm{mL} .{ }^{\mathrm{f}}$ Units: $\mathrm{ng} / \mathrm{dL} .{ }^{\mathrm{g}}$ Units: $\mu \mathrm{g} / \mathrm{dL} .{ }^{\mathrm{I}}$ BPS, BPF, TCC and EPB had the lowest sample sizes because they were added to the biomarker assay at mid-study. At 16-20 weeks, these four urinary biomarkers had $N=295$. At $24-28$ weeks, these four urinary biomarkers had $N=198$

was moderately correlated with 2,4-dichlorophenol (Spearman $r=0.5, p<0.001$ ), but not with 2,5-dichlorophenol (Spearman $r=-0.03$ ). BPA, BPS and BPF showed low correlation (Spearman $\mathrm{r}=0.11-0.21, p<0.001$ ).

Results from LMMs and MLRs are described in detail below by biomarker (Tables 3, Additional file 1: Table S1 and S2, and Additional file 2). There were few differences between most adjusted and unadjusted models, with the exception of associations with CRH. MPB and PPB were associated with $\mathrm{CRH}$ in our unadjusted models, but in the adjusted models, these associations disappeared, and $\mathrm{CRH}$ was associated with BPS and TCS. A further analysis of $\mathrm{CRH}$ concentrations across the covariate levels did not reveal any large differences to report.
There were no associations between 2,4-dichlorophenol and 2,5-dichlorophenol with hormones in LMMs. An IQR increase in 2,4-dichlorophenol was associated with a $10 \%$ increase in CRH at 24-28 weeks [9.66\% change in hormone per IQR change in the biomarker/ percent change in hormone at detectable biomarker concentrations $(\% \Delta)$; $95 \%$ CI: $0.67,19.45]$, and a suggestive $2 \%$ decrease in T3 at $16-20$ weeks $(\% \Delta-2.22$ $95 \%$ CI $-4.55,0.10)$.

Associations across the bisphenols differed, and BPS had the strongest associations in LMM models. BPS was associated with an $11 \%$ decrease in CRH (\% $\Delta-11.35$; 95\% CI: $-18.71,-3.33)$, and this association was stronger at $16-20$ weeks gestation. At this time point, BPS was 


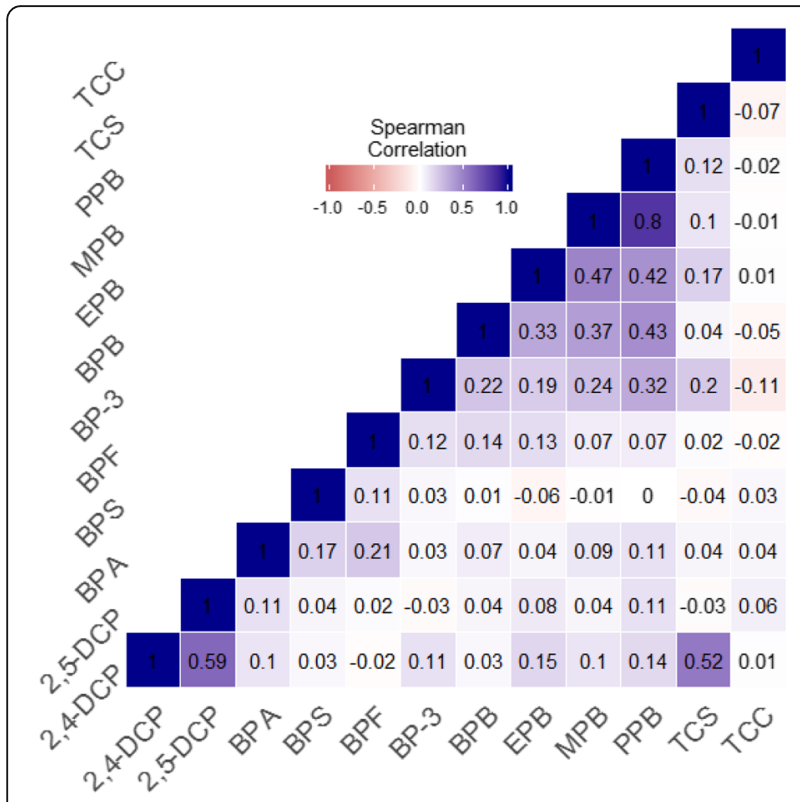

Fig. 1 Heat map of Spearman correlations between unlogged urinary triclocarban, phenols and parabens. Biomarkers concentrations were adjusted for urinary dilution. 2,4-DCP: 2,4-dichlorophenol; 2,5-DCP: 2,5-dichlorophenol; BP-3: Benzophenone; TCS: Triclosan; TCC:

Triclocarban; EPB: ethylparaben; MPB: Methylparaben; BPB: Butylparaben; PPB: Propylparaben

additionally associated with a $12 \%$ decrease in TSH $(\% \Delta$ -11.93; 95\% CI: $-22.49,0.07)$. BPF was associated with a $3 \%$ increase in FT4 (\% 2.76 ; 95\% CI: 0.29, 5.22), and this association was also stronger at 16-20 weeks. BPA, on the other hand, had stronger associations at 24-28 weeks gestation. BPA was associated with a $17 \%$ decrease in testosterone, and 2-4\% increases in FT4 and T3 at 24-28 weeks [(\% -17.37; 95\% CI: $-26.7,-6.87)$; $(\% \Delta$ 2.38; 95\% CI: 0.04, 4.72); (\% $\Delta 4.33,95 \%$ CI: 0.11 , 8.55), respectively]. The increase in FT4 and T3 in relation to BPA was in line with our a priori hypothesis Benzophenone-3 was not significantly associated with any hormones.

Triclocarban was associated with a number of thyroid hormones and SHBG. An IQR increase in triclocarban is associated with a $4 \%$ increase in T3 $(\% \Delta 4.08 ; 95 \% \mathrm{CI}$ : $1.18,6.98)$, a $5 \%$ increase in the $\mathrm{T} 3 / \mathrm{T} 4$ ratio $(\% \Delta 4.67$; $95 \%$ CI: $1.24,10.10)$, a suggestive $10 \%$ decrease in $\mathrm{TSH}$ (\% $\Delta-10.12$; $95 \% \mathrm{CI}:-19.47,0.32)$, and a $10 \%$ decrease in SHBG at 24-28 weeks (\% -9.71 ; 95\% CI: $-19.1,-0.27)$.

Triclosan was associated with an increase in a number of reproductive hormones, however most were only suggestive with $p$ values between 0.05 and 0.10 . This includes a $9 \%$ increase in CRH (\% $\triangle 9.20 ; 95 \% \mathrm{CI}:-0.97$, 20.42), a $7 \%$ increase in testosterone $(\% \Delta 7.13$; $95 \% \mathrm{CI}$ : $-0.60,15.5)$, and $10-13 \%$ increases in progesterone and estriol at 24-28 weeks [(\% $\Delta$ 9.72, 95\% CI: $-1.27,21.9)$; (\% $\Delta$ 13.2; 95\% CI: 2.34, 25.2), respectively]. In addition, triclosan was associated with a 5.8\% decrease in T3 at 24-28 weeks; this finding was in line with our a priori hypothesis.

IQR increases in butylparaben, methylparaben and propylparaben were associated with a decrease in SHBG [(\% -5.27; 95\% CI:-9.40, - 1.14); (\% -3.53 ; 95\% CI: -7.37, 0.31); (\% -3.74; 95\% CI: $-7.76,0.27)$, respectively]. Methylparaben was also associated with decreases in reproductive hormones, including an $8 \%$ decrease in estriol, a suggestive 3\% increase in the progesterone/estriol ratio, and a suggestive decrease in testosterone at 16-20 weeks [(\% $\Delta-7.76 ; 95 \%$ CI: $-15.4,0.61) ;(\% \Delta$ 3.14; 95\% CI: $-2.95,9.61)$; (\% $\Delta-6.77$; 95\% CI: $-13.13,0.29)$, respectively]. Conversely, an IQR increase in propylparaben was associated with a $9-10 \%$ increase in progesterone and estriol at 24-28 weeks [(\% $\Delta$ 9.67; 95\% CI: -1.30 , 21.85); (\% $\Delta .92$; 95\% CI: -1.56, 20.52)]. Interaction terms between study visit:methylparaben and propylparaben had $p$ values $<0.05$ in models regressed against estriol. We expected to see a decrease in thyroid hormones in relation to methyl- and propyl- paraben, but only observed a decrease in TSH in association with methylparaben, particularly at $16-20$ weeks $(\% \Delta-11.69 ; 95 \%$ CI: -21.97, - 0.06). The decrease in TSH could indicate an increase in circulating thyroid hormones, in contrast to our hypothesis.

\section{Discussion}

Associations differed by exposure biomarker and hormone, and there was little consistency within chemical classes with the exception of some parabens. There was evidence of a decrease up to $6 \%$ in $\mathrm{T} 3$ in association with 2,4-dichlorophenol, BPA and triclosan, whereas triclocarban was associated with a $4 \%$ increase in T3. In the case of bisphenols, BPS was more strongly related to decreases in hormones at 16-20 weeks, and BPA had stronger negative relationships at 24-28 weeks. Triclosan was associated with general increases in reproductive hormones of approximately 10\%, and triclocarban was associated with $5-10 \%$ changes in thyroid hormones. Parabens were associated with a decreased level of SHBG.

While there may be structural similarities between BPA, BPS and BPF, the structural variations may be sufficient to alter receptor-binding affinities across the bisphenols [37]; therefore, the biological effects may vary among the bisphenols. To this, we found that the earlier time point (16-20 weeks gestation) may be a more vulnerable time of exposure to BPS and BPF, in contrast to the stronger relationships observed at the 24-28 weeks with respect to BPA. Our results were somewhat consistent with results from previous studies. BPA has been suspected to interfere with thyroid hormones, as evidenced by several epidemiological studies. We observed an increase in FT4 and T3, which was consistent with two 
Table 3 Results of the adjusted LMMs regressing hormones versus exposure biomarkers

\begin{tabular}{|c|c|c|c|c|c|c|c|}
\hline & & $\mathrm{CRH}$ & SHBG & Testosterone & Progesterone & Estriol & $\begin{array}{l}\text { Progesterone/ } \\
\text { Estriol Ratio }\end{array}$ \\
\hline \multirow[t]{2}{*}{$\begin{array}{l}2,4- \\
\text { DCP }\end{array}$} & $\begin{array}{l}\% \Delta / \\
\text { IQR }\end{array}$ & $5.30(-2.81,14.08)$ & $2.06(-1.49,5.61)$ & $3.26(-3.01,9.94)$ & $1.60(-3.42,6.87)$ & $-1.93(-7.54,4.01)$ & $3.58(-1.85,10.16)$ \\
\hline & $p$ & 0.21 & 0.26 & 0.32 & 0.54 & 0.52 & 0.24 \\
\hline \multirow[t]{2}{*}{$\begin{array}{l}2,5- \\
\mathrm{DCP}\end{array}$} & $\begin{array}{l}\% \Delta / \\
\text { IQR }\end{array}$ & $3.89(-3.29,11.61)$ & $0.96(-2.25,4.17)$ & $1.75(-3.85,7.69)^{a}$ & $-0.46(-4.79,4.07)$ & $-2.21(-7.12,2.96)$ & $1.35(-3.46,6.82)$ \\
\hline & $p$ & 0.30 & 0.56 & 0.55 & 0.84 & 0.40 & 0.61 \\
\hline \multirow[t]{2}{*}{ BPA } & $\begin{array}{l}\% \Delta / \\
\mathrm{IQR}\end{array}$ & $3.68(-4.21,12.22)$ & $-0.22(-3.60,3.15)$ & $\begin{array}{l}-4.19(-9.64 \\
1.59)^{\mathrm{a}}\end{array}$ & $-3.50(-8.16,1.39)$ & $-2.18(-7.78,3.78)$ & $-1.55(-6.18,5.01)$ \\
\hline & $p$ & 0.37 & 0.90 & 0.15 & 0.16 & 0.47 & 0.60 \\
\hline \multirow[t]{2}{*}{$\mathrm{BPF}^{\mathrm{b}}$} & $\begin{array}{l}\% \Delta / \\
\text { IQR }\end{array}$ & $3.90(-9.72,19.57)$ & $-2.97(-8.04,2.11)$ & $0.33(-8.89,10.49)$ & $\begin{array}{l}-1.33(-23.9 \\
13.78)\end{array}$ & $3.84(-6.40,15.21)$ & $\begin{array}{l}-4.65(-13.44 \\
5.05)\end{array}$ \\
\hline & $p$ & 0.60 & 0.26 & 0.95 & 0.76 & 0.48 & 0.34 \\
\hline \multirow[t]{2}{*}{ BPS } & $\begin{array}{l}\% \Delta / \\
\text { IQR }\end{array}$ & $\begin{array}{l}-11.35(-18.71,- \\
3.33)\end{array}$ & $-0.56(-4.37,3.25)$ & $2.54(-3.5,8.97)$ & $-4.38(-9.49,1.02)$ & $-2.05(-8.16,4.47)$ & $-2.96(-7.85,3.85)$ \\
\hline & $p$ & $0.008^{* *}$ & 0.77 & 0.42 & 0.11 & 0.53 & 0.34 \\
\hline \multirow[t]{2}{*}{ BP-3 } & $\begin{array}{l}\% \Delta / \\
\mathrm{IQR}\end{array}$ & $-0.04(-7.96,8.57)$ & $1.10(-2.61,4.82)$ & $-0.51(-6.8,6.21)$ & $0.46(-4.62,5.81)$ & $-0.91(-6.66,5.18)$ & $1.81(-3.60,8.42)$ \\
\hline & $p$ & 0.99 & 0.56 & 0.88 & 0.86 & 0.76 & 0.56 \\
\hline \multirow[t]{2}{*}{ TCC } & $\begin{array}{l}\% \Delta / \\
\text { IQR }\end{array}$ & $-3.69(-14.5,8.50)$ & $\begin{array}{l}-4.54(-10.03 \\
0.94)\end{array}$ & $5.18(-3.4,14.51)$ & $\begin{array}{l}-3.22(-10.13 \\
4.21)\end{array}$ & $0.36(-7.93,9.39)$ & $-3.75(-8.64,7.7)$ \\
\hline & $p$ & 0.54 & 0.11 & 0.25 & 0.39 & 0.94 & 0.39 \\
\hline \multirow[t]{2}{*}{ TCS } & $\begin{array}{l}\% \Delta / \\
\text { IQR }\end{array}$ & $9.20(-0.97,20.42)$ & $2.81(-1.46,7.08)$ & $7.13(-0.60,15.5)$ & $2.84(-3.2,9.25)^{a}$ & $4.16(-3.07,11.93)^{\mathrm{a}}$ & $0.31(-5.8,8.4)$ \\
\hline & $p$ & $0.08^{*}$ & 0.20 & $0.07^{*}$ & 0.37 & 0.27 & 0.93 \\
\hline \multirow[t]{2}{*}{$E P B^{b}$} & $\begin{array}{l}\% \Delta / \\
\mathrm{IQR}\end{array}$ & $1.52(-11.55,16.52)$ & $-1.93(-8.14,4.29)$ & $\begin{array}{l}5.11 \\
a\end{array}$ & $\begin{array}{l}-2.41(-10.62 \\
6.56)\end{array}$ & $-1.92(-11.4,8.58)$ & $\begin{array}{l}-0.77(-10.22 \\
9.67)\end{array}$ \\
\hline & $p$ & 0.83 & 0.55 & 0.32 & 0.59 & 0.71 & 0.88 \\
\hline \multirow[t]{2}{*}{ BPB } & $\begin{array}{l}\% \Delta / \\
\text { IQR }\end{array}$ & $-1.86(-10.64,7.8)$ & $-5.27(-9.4,-1.14)$ & $-6.77(-13.3,0.29)$ & $-3.65(-9.11,2.14)$ & $-5.18(-11.45,1.52)$ & $1.96(-4.9,8.52)$ \\
\hline & $p$ & 0.70 & $0.01^{* *}$ & $0.06^{*}$ & 0.21 & 0.13 & 0.58 \\
\hline \multirow[t]{2}{*}{ MPB } & $\begin{array}{l}\% \Delta / \\
\text { IQR }\end{array}$ & $5.88(-3.0,15.59)$ & $-3.53(-7.37,0.31)$ & $-4.41(-10.68,2.3)$ & $0.03(-5.29,5.64)$ & $-2.50(-8.6,4.01)^{\mathrm{a}}$ & $2.64(-3.06,9.74)$ \\
\hline & $p$ & 0.20 & $0.07^{*}$ & 0.19 & 0.99 & 0.44 & 0.43 \\
\hline \multirow[t]{3}{*}{ PPB } & $\begin{array}{l}\% \Delta / \\
\text { IQR }\end{array}$ & $4.82(-4.48,15.02)$ & $-3.74(-7.76,0.27)$ & $\begin{array}{l}-3.54(-10.14 \\
3.54)\end{array}$ & $2.35(-3.55,8.6)$ & $-0.63(-7.36,6.58)^{a}$ & $3.65(-2.36,11.66)$ \\
\hline & $p$ & 0.32 & $0.07^{*}$ & 0.32 & 0.44 & 0.86 & 0.31 \\
\hline & & TSH & FT4 & T3 & T4 & T3/T4 ratio & \\
\hline \multirow[t]{2}{*}{$\begin{array}{l}2,4- \\
\mathrm{DCP}\end{array}$} & $\begin{array}{l}\% \Delta / \\
\text { IQR }\end{array}$ & $4.80(-2.58,12.74)$ & $0.21(-1.19,1.60)$ & $-1.58(-3.58,0.42)$ & $-0.79(-2.71,1.13)$ & $-1.16(-4.86,1.33)$ & \\
\hline & $p$ & 0.21 & 0.77 & 0.12 & 0.42 & 0.31 & \\
\hline \multirow[t]{2}{*}{$\begin{array}{l}2,5- \\
D C P\end{array}$} & $\begin{array}{l}\% \Delta / \\
\text { IQR }\end{array}$ & $4.63(-2.08,11.79)$ & $0.82(-0.43,2.07)$ & $-0.55(-2.36,1.26)$ & $0.51(-1.22,2.24)$ & $-1.38(-4.64,1.06)$ & \\
\hline & $p$ & 0.18 & 0.2 & 0.56 & 0.57 & 0.18 & \\
\hline \multirow[t]{2}{*}{ BPA } & $\begin{array}{l}\% \Delta / \\
\text { IQR }\end{array}$ & $-0.28(-6.99,6.91)$ & $0.00(-1.36,1.36)$ & $2.10(0.22,3.99)$ & $0.69(-1.13,2.51)$ & $1.46(-2.23,3.52)$ & \\
\hline & $p$ & 0.94 & 1 & $0.03^{* *}$ & 0.46 & 0.19 & \\
\hline $\mathrm{BPF}^{\mathrm{b}}$ & $\begin{array}{l}\% \Delta / \\
\text { IQR }\end{array}$ & $7.29(-4.59,20.64)$ & $2.76(0.29,5.22)^{a}$ & $-1.22(-4.34,1.90)$ & $1.84(-1.37,5.04)$ & $-2.50(-6.47,1.47)$ & \\
\hline
\end{tabular}


Table 3 Results of the adjusted LMMs regressing hormones versus exposure biomarkers (Continued)

\begin{tabular}{|c|c|c|c|c|c|c|}
\hline & $p$ & 0.24 & $0.03^{* *}$ & 0.45 & 0.26 & 0.22 \\
\hline \multirow[t]{2}{*}{ BPS } & $\begin{array}{l}\% \Delta / \\
\text { IQR }\end{array}$ & $-1.01(-8.12,6.66)$ & $-0.07(-1.6,1.46)$ & $0.14(-1.89,2.18)$ & $0.04(-1.97,2.05)$ & $0.58(-2.47,2.9)$ \\
\hline & $p$ & 0.79 & 0.93 & 0.89 & 0.97 & 0.64 \\
\hline \multirow[t]{2}{*}{ BP-3 } & $\begin{array}{l}\% \Delta / \\
\text { IQR }\end{array}$ & $-5.89(-12.87,1.65)$ & $-0.40(-1.85,1.04)$ & $-1.47(-3.56,0.63)$ & $-1.37(-3.35,0.62)$ & $-0.21(-5.33,1.26)$ \\
\hline & $p$ & 0.13 & 0.59 & 0.17 & 0.18 & 0.86 \\
\hline \multirow[t]{2}{*}{ TCC } & $\begin{array}{l}\% \Delta / \\
\text { IQR }\end{array}$ & $-10.12(-19.47,0.32)$ & $-0.61(-2.76,1.55)$ & $4.08(1.18,6.98)$ & $-0.65(-3.53,2.23)$ & $4.67(-1.37,6.65)$ \\
\hline & $p$ & $0.06^{*}$ & 0.58 & $0.007^{* *}$ & 0.66 & $0.01^{* *}$ \\
\hline \multirow[t]{2}{*}{ TCS } & $\begin{array}{l}\% \Delta / \\
\text { IQR }\end{array}$ & $0.57(-7.74,9.63)$ & $-0.74(-2.45,0.96)$ & $-1.97(-4.36,0.41)$ & $-1.60(-3.9,0.69)$ & $-0.15(-2.69,4.55)$ \\
\hline & $p$ & 0.9 & 0.39 & 0.11 & 0.17 & 0.91 \\
\hline \multirow[t]{2}{*}{$E P B^{b}$} & $\begin{array}{l}\% \Delta / \\
\text { IQR }\end{array}$ & $-6.78(-17.6,5.46)$ & $-0.45(-2.9,2.0)$ & $-0.76(-4.10,2.58)$ & $-0.03(-3.29,3.24)$ & $-1.68(-5.66,2.30)$ \\
\hline & $p$ & 0.27 & 0.72 & 0.66 & 0.99 & 0.41 \\
\hline \multirow[t]{2}{*}{ BPB } & $\begin{array}{l}\% \Delta / \\
\text { IQR }\end{array}$ & $-4.88(-12.62,3.54)$ & $1.10(-0.54,2.74)$ & $0.70(-1.61,3.02)$ & $1.74(-0.49,3.96)$ & $-1.56(-7.01,-0.26)$ \\
\hline & $\mathrm{p}$ & 0.25 & 0.19 & 0.55 & 0.13 & 0.24 \\
\hline \multirow[t]{2}{*}{ MPB } & $\begin{array}{l}\% \Delta / \\
\text { IQR }\end{array}$ & $-6.92(-13.91,0.64)^{a}$ & $0.77(-0.76,2.29)$ & $-0.39(-2.53,1.76)$ & $1.02(-1.04,3.09)$ & $-1.78(-4.64,1.53)$ \\
\hline & $p$ & $0.07^{*}$ & 0.33 & 0.73 & 0.33 & 0.15 \\
\hline \multirow[t]{2}{*}{ PPB } & $\begin{array}{l}\% \Delta / \\
\mathrm{IQR}\end{array}$ & $-6.29(-13.6,1.64)$ & $0.81(-0.8,2.42)$ & $0.21(-2.02,2.45)$ & $0.65(-1.52,2.81)$ & $-0.67(-3.89,2.63)$ \\
\hline & $p$ & 0.12 & 0.32 & 0.85 & 0.56 & 0.6 \\
\hline
\end{tabular}

2,4-DCP: 2,4-dichlorophenol; 2,5-DCP: 2,5-dichlorophenol; BP-3: Benzophenone; TCS: Triclosan; TCC: Triclocarban; EPB: ethylparaben; MPB: Methylparaben; BPB: Butylparaben; PPB: Propylparaben

Results converted to \% change in hormone per IQR change in biomarker concentration

* represents a $p$ value below 0.10 , and ${ }^{* *}$ represents a $p$ value below 0.05 ; a Significant interaction $(p<0.05)$ between urinary biomarker*visit; $b$ Dichotomous variable

Models adjusted for specific gravity, study visit, body mass index (BMI) at the first study visit, maternal age, the number of hours of second-hand smoking exposure per day, and a socio-economic variable

previous studies our group conducted in a preliminary analysis in the PROTECT cohort, and a cohort of pregnant women in Boston, MA with four repeated measures during pregnancy $[38,39]$. Two cross-sectional studies in the United States $(N=249$ and 476 women) also looked at the association between maternal BPA and thyroid hormones during gestation [40, 41]. The only significant association reported was between maternal urinary BPA and a decrease in T4 [40], which we did not observe in the present study. A decrease in T4 could be indicative of an increase in FT4, in the case of thyroxine becoming less bound to thyroxine-binding globulin, however, the associations between BPA and T4 in the current study had $p$ values ranging from $0.51-0.93$. Furthermore, we did not observe a relationship between BPA and TSH that was reported in the Boston cohort study [42], and among adults from the Korean National Environmental Health Survey [43].

One of the strongest associations we observed was the $17 \%$ decrease in testosterone in relation to BPA. This is the first study that explores this association in pregnant women, and there is little correlation between maternal and fetal testosterone levels [44]. However, a decrease in testosterone was identified in an in vitro study on TM3 murine Leydig with BPA exposure [45], in the F2 generation after in-utero BPA exposure in mice [46], and in-utero BPA concentrations in young boys aged 8-14 [47]. These associations provide further evidence in support of our finding. Although the role of maternal testosterone in gestation is still unclear, evidence points to androgens playing an essential role in myometrial relaxation, cervical ripening and initiating parturition [48]. Therefore, BPA, via reduced testosterone, could increase gestational age, which we previously observed in this cohort [49]. Additionally, maternal testosterone has a role in gender role behaviors [50], indicating that maternal testosterone may impact fetal development.

No human studies have previously investigated the associations between triclocarban, phenols and parabens on $\mathrm{CRH}$ during pregnancy; however, $\mathrm{CRH}$ plays an important role in gestation. Maternal CRH levels during 
pregnancy largely originate from gestational tissues [51]. Evidence suggests $\mathrm{CRH}$ inhibits immune rejection processes by killing activated $T$ cells [52], plays an important role in determining time of parturition, and an increase in $\mathrm{CRH}$ has been associated with the onset of miscarriage and preeclampsia [53-57]. CRH receptor expression is regulated by estrogen, and $\mathrm{CRH}$ gene expression in the placenta is mediated by ER- $\alpha[58,59]$. Given the endocrine disrupting potential of bisphenols via estrogen receptors [60], associations between $\mathrm{CRH}$ and bisphenols (and potentially other phenols and parabens) could be important to consider in pregnancy studies. Animal and in vitro studies showed an increase in $\mathrm{CRH}$ with exposure to BPA and BPS, contrary to our results of an inverse relationship between $\mathrm{CRH}$ and BPS. BPA increased plasma concentrations of $\mathrm{CRH}$ in pregnant mice [61] and $\mathrm{CRH}$ levels in human placenta primary trophoblast cells [62]. The differences in our results could be in part due to the unique role $\mathrm{CRH}$ plays in human pregnancies, as compared to animals [63].

Triclosan was suggestively associated with select hormones, but none reached statistical significance, including an increase in testosterone, an increase in $\mathrm{CRH}$ at 16-20 weeks gestation, and a decrease in T3 at 24-28 weeks gestation. There was a similar decrease in T3 with increased urinary triclosan concentrations in the Boston cohort, albeit the associations were stronger earlier in pregnancy, in contrast to our stronger associations at the later visit in the current study [39]. While larger human studies with more statistical power may be needed, the decrease in T3 in association with triclosan is consistent with animal studies [64], including in pregnant rats [65] and pregnant mice [66, 67], perhaps due to triclosan's structural similarities to thyroid hormones [64]. Animal studies also report a decrease in T4 with triclosan exposure, including rat and mice dams [65-75], but we did not find evidence of this in humans. Other population studies found no associations between triclosan and thyroid hormones [76-78], although there was evidence of vulnerable time points during gestation [76, 77]. Interestingly, a study in pregnant rats showed that the greatest accumulation of triclosan was in the placenta, indicating that pregnancy may be a sensitive time period for triclosan exposure [79]. Alternatively, maternal serum TSH and FT4 levels at $>28$ weeks gestation (obtained from medical records) were negatively associated with urinary triclosan at 38 weeks gestation [80]. The differences in our results could be explained by the differences in the study population, exposure biomarker concentrations, and differences in the pregnancy time points examined.

No studies have looked at the effect of triclosan on maternal testosterone and CRH during pregnancy in humans. However, in contrast to our results, triclosan was found to reduce testosterone levels in male rats [81], and in pregnant rats [79]. An excess of maternal testosterone has been associated with restricted fetal growth [82], as well as an increased chance of developing Alzheimer disease [83] and anxiety like symptoms in the offspring.

Triclocarban was associated with thyroid hormone changes. We observed an increase in T3 and a decrease in TSH in association with triclocarban, which is in line with the negative feedback loop in maintaining thyroid hormone homeostasis. We also observed a decrease in SHBG. SHBG levels tend to rise with thyroid hormones, so this observed pattern was unexpected. This could be due to factors influencing the relationship between thyroid hormone and SHBG levels that have not been accounted for in the present study. Our previous Boston study also reported a negative association between triclocarban and TSH, but a negative association with T3. Triclocarban concentrations in this cohort were much higher than the exposure levels found in the Boston cohort. In fact, the triclocarban concentrations observed in PROTECT are 37 times larger than the concentration observed in NHANES women of reproductive age [84]. This difference in exposure levels may explain the differences in the associations observed.

All parabens were generally negatively associated with SHBG. In contrast to our current findings, our previous preliminary analysis in the PROTECT cohort showed that methylparaben was positively associated with SHBG [26]. However, the current study has a much larger sample size. Associations between parabens and some hormones appeared to be dependent on the timing of exposure. Associations between methylparaben and propylparaben and estriol changed direction from a negative association at $16-20$ weeks to a positive association at 24-28 weeks gestation. We observed a similar change in direction in our preliminary analyses between methylparaben and propylparaben with estradiol [26]. Although not statistically significant, associations between methylparaben and propylparaben with progesterone followed a similar pattern to that of estriol. Given that the population urinary levels of methylparaben and propylparaben remained consistent between the two time points, the similar change of direction observed in associations with methylparaben and propylparaben in both of our previous analyses, and the significant interaction term between these parabens and visit in association with estriol, this lends confidence that these observations may not be occurring by chance and may be detected in future larger studies. The strong correlation between propyl- and methylparaben could indicate that their associations with estriol are being driven by only one of the parabens. However, given the differences in the associations between these two parabens and all hormones, there do seem to be unique relationships between the exposure and hormone levels. No previous studies have looked at the effect of parabens on estriol, 
SHBG or CRH; however, evidence suggests parabens have ER- $\beta$ agonistic activity [85], and stimulate progesterone mRNA expression via ER- $\alpha$ signaling $[86,87]$. This could suggest a potential mechanism by which reproductive hormone levels could be directly or indirectly altered in response to paraben exposure.

The present study also showed a general decrease in TSH in association with parabens, but only methylparaben reached a significant association with TSH. Additionally, methylparaben and propylparaben were associated with a decrease in the T3/T4 ratio, particularly at 24-28 weeks gestation. Results from our Boston cohort also showed a decrease in T3/T4 ratio, as well as T3, at median 26 weeks gestation [88]. In other research, human and animal studies reported a decrease in T4 and FT4 with paraben exposure in females $[78,89]$, and two small studies in men found no associations between parabens and thyroid hormones [90, 91]. The difference in the results is likely due to the different study populations; none of those studies looked specifically at prenatal exposure.

Our study had several limitations. We did not have data on the iodine status of the women; deficiency in this element could affect thyroid hormone function. However, iodine may act as mechanistic intermediate exposure between the exposure and thyroid hormone, and controlling for iodine status could lead to bias [92]. Furthermore, iodine had no effect on the associations between phenols and thyroid hormones in our previous study of NHANES data [78]. We also did not have data on thyroperoxidase antibodies nor human chorionic gonadotropin (hCG), which could potentially affect thyroid function as well [93, 94]. While data at two time points is a great improvement from the more common cross-sectional study design, the two time points may not be sufficient to understand the potential influence of these biomarkers on maternal hormones. The relatively high variation in urinary concentrations of the target biomarkers (particularly BPA) over time may also introduce potential bias stemming from random measurement error. Given the multiple comparisons conducted, there is a chance of Type I error, and caution must be used when interpreting our findings. Finally, although one of the strengths of the present study is our ability to investigate the relationships between these chemicals and hormone levels in a vulnerable population, our study population was based in a population in Puerto Rico of lower income who also had higher urinary concentrations of some of the exposure biomarkers; therefore, the results may not be fully generalizable to other populations.

Our study also had many strengths. Our robust sample size, and the collection of exposure biomarkers and hormone data at two time points during pregnancy helps account for the biomarkers' short lifespan in the body, and the varying levels of hormones throughout pregnancy. The repeated measures allow for the control of intra-individual variability, and increases statistical power. We were also able to explore potential windows of susceptibility for these associations.

Additionally, we were able to compare our results from this analysis to our own analyses that employed similar statistical methods in two other data sets, namely LMMs to capture biomarkers at various time points and allow subject-specific intercepts. While there were many similarities in the results across the three analyses, the differences in results may point to the importance of outside factors that may not be captured in our models that alter the associations between these chemicals and endocrine disruption through interaction with the chemicals. These outside factors could include other endocrine-altering variables, such as exposure to other unaccounted for chemicals, maternal stress, genetic, epigenetic, or other differences. It is imperative that future studies look beyond the association between a single chemical and singe hormone, and explore potential interactions with chemical exposure.

\section{Conclusion}

Our results provide suggestive human evidence for associations between select biomarkers with maternal thyroid and reproductive hormones during gestation. Of note, we report negative associations between parabens and SHBG, a negative association between BPS and $\mathrm{CRH}$, and associations between triclocarban and triclosan with reproductive and thyroid hormones. Our stratified analyses show that some associations may be stronger at certain time points during pregnancy. Further studies in larger populations and with more repeated measures across pregnancy to will be useful to confirm our findings, and better understand if and how these hormone changes may affect downstream maternal and infant health outcomes.

\section{Additional file}

Additional file 1: Table S1. Results of the adjusted MLRs regressing reproductive hormones versus exposure biomarkers by visit. Table S2. Results of the adjusted MLRs regressing thyroid hormones versus exposure biomarkers by visit. Table S3. Result comparison between the common exposure biomarkers and hormones. (DOCX $32 \mathrm{~kb}$ )

Additional file 2: Adjusted multiple linear regressions of hormones versus urinary concentrations of biomarkers stratified by study visit. Visit 1: 16-20 weeks; Visit 3: 24-28 weeks. EPB and BPF are categorical variables. * represents at least one marginal association between the urinary concentration and the hormone across the four time points. ${ }^{* *}$ represents at least one significant association between the urinary biomarker concentration and the hormone across the four time points. BPF and EPB were dichotomous variables. 2,4-DCP: 2,4-dichlorophenol; 2,5-DCP: 2,5-dichlorophenol; BP-3: Benzophenone; TCS: 
Triclosan; TCC: Triclocarban; EPB: ethylparaben; MPB: Methylparaben; BPB: Butylparaben; PPB: Propylparaben (DOCX 664 kb)

\section{Abbreviations}

BMI: Body mass index; BPA: Bisphenol-A; BPF: Bisphenol-F; BPS: Bishphenol-S; CDC: Centers for Disease Control and Prevention; Cl: Confidence intervals; CRH: Corticotropin-releasing hormone; FT4: Free thyroxine; hCG: Human chorionic gonadotropin; LMM: Linear Mixed Models; LOD: Limit of detection; MLR: Multiple linear regression; NHANES: National Health and Nutrition Examination Survey; PROTECT: Puerto Rico Testsite for Exploring Contamination Threats; SG: Specific gravity; SHBG: Sex-hormone-binding globulin; T3: Total triiodothyronine; T4: Total thyroxine; TSH: Thyroidstimulating hormone

\section{Acknowledgements}

We gratefully acknowledge Antonia Calafat and Xiaoyun Ye at the Centers for Disease Control and Prevention for analysis of urinary phenol, paraben and triclocarban concentrations. We would like to thank and D. McConnell of the CLASS Lab at University of Michigan for assistance in hormone analysis.

\section{Funding}

This work was supported by the National Institute of Environmental Health Sciences, National Institutes of Health (Grants P42ES017198, P50ES026049, and UG3OD023251). Funding for Ferguson KK was provided by the Intramural Research Program of the National Institute of Environmental Health Sciences, $\mathrm{NIH}$. The funding sources had no involvement in the study design, collection, analysis \& interpretation of data or writing of the report. The findings and conclusions in this report are those of the authors and do not necessarily represent the official position of the Centers for Disease Control and Prevention. Use of trade names is for identification only and does not imply endorsement by the CDC, the Public Health Service, or the US Department of Health and Human Services.

\section{Availability of data and materials}

Datasets analyzed for the current study are not publicly available because they contain sensitive and protected health information on our participants.

\section{Authors' contributions}

AMA: data analysis, AMA \& JDM: data interpretation and drafting of document, KKF: data cleaning, JDM, ANA \& JFC: conception and design, ZYR: acquisition of data, BM: methodology advisor; AMC: data measurement. All authors read and approved the final manuscript.

\section{Ethics approval and consent to participate}

This study was approved by the research and ethics committees of the University Of Michigan School Of Public Health, University of Puerto Rico, Northeastern University, and the University of Georgia. All study participants provided full informed consent prior to participation.

\section{Consent for publication}

Not applicable.

\section{Competing interests}

The authors declare that they have no competing interests. The involvement of the Centers for Disease Control and Prevention (CDC) laboratory did not constitute engagement in human subjects research.

\section{Publisher's Note}

Springer Nature remains neutral with regard to jurisdictional claims in published maps and institutional affiliations.

\section{Author details}

'Department of Environmental Health Sciences, University of Michigan School of Public Health, Room 1835 SPH I, 1415 Washington Heights, Ann Arbor, MI 48109-2029, USA. Epidemiology Branch, Intramural Research Program, National Institute of Environmental Health Sciences, Durham, USA. ${ }^{3}$ Graduate School of Public Health, Medical Sciences Campus, University of Puerto Rico, San Juan, PR, USA. ${ }^{4}$ Department of Biostatistics, University of Michigan School of Public Health, Ann Arbor, MI, USA. ${ }^{5}$ College of Engineering, Northeastern University, Boston, MA, USA. ${ }^{6}$ Centers for Disease
Control and Prevention, Atlanta, GA, USA. ${ }^{7}$ College of Public Health, University of Georgia, Athens, GA, USA.

Received: 28 June 2018 Accepted: 28 February 2019

Published online: 02 April 2019

\section{References}

1. Karpuzoglu E, Holladay SD, Gogal RM. Parabens: potential impact of lowaffinity estrogen receptor binding chemicals on human health. J Toxicol Environ Health B Crit Rev. 2013;16:321-35

2. Peretz J, Vrooman L, Ricke WA, Hunt PA, Ehrlich S, Hauser R, et al. Bisphenol a and reproductive health: update of experimental and human evidence, 2007-2013. Environ Health Perspect. 2014;122:775-86.

3. Rochester JR. Bisphenol A and human health: A review of the literature. Reprod Toxicol. 2013:42:132-55.

4. Rochester JR, Bolden AL. Bisphenol S and F: a systematic review and comparison of the hormonal activity of bisphenol a substitutes. Environ Health Perspect. 2015;123:643-50.

5. Tang R, Chen M-J, Ding G-D, Chen X-J, Han X-M, Zhou K, et al. Associations of prenatal exposure to phenols with birth outcomes. Environ Pollut Barking Essex. 2013;178:115-20.

6. Lassen TH, Frederiksen H, Kyhl HB, Swan SH, Main KM, Andersson A-M, et al. Prenatal Triclosan Exposure and Anthropometric Measures including Anogenital Distance in Danish Infants. Environ Health Perspect. 2016;124(8): 1261-8. https://doi.org/10.1289/ehp.1409637.

7. Vernet C, Pin I, Giorgis-Allemand L, Philippat C, Benmerad M, Quentin J, et al. In Utero Exposure to Select Phenols and Phthalates and Respiratory Health in Five-Year-Old Boys: A Prospective Study. Environ Health Perspect. 2017;125(9):097006. https://doi.org/10.1289/EHP1015.

8. Gutiérrez-Torres DS, Barraza-Villarreal A, Hernandez-Cadena L, EscamillaNuñez C, Romieu I. Prenatal exposure to endocrine disruptors and Cardiometabolic risk in preschoolers: a systematic review based on cohort studies. Ann Glob Health. 2018:84:239-49.

9. Centers for Disease Control and Prevention. Fourth National Report on Human Exposure to Environmental Chemicals, Updated Tables, January 2017 [Internet]. Atlanta, GA: Centers for Disease Control and Prevention (CDC); 2017 . Available from: https://www.cdc.gov/exposurereport/pdf/ FourthReport_UpdatedTables_Volume1_Jan2017.pdf. Accessed 9 Mar 2019.

10. Boberg J, Taxvig C, Christiansen S, Hass U. Possible endocrine disrupting effects of parabens and their metabolites. Reprod Toxicol Elmsford N. 2010; 30:301-12.

11. Dodson RE, Nishioka M, Standley LJ, Perovich LJ, Brody JG, Rudel RA. Endocrine disruptors and asthma-associated chemicals in consumer products. Environ Health Perspect. 2012;120:935-43.

12. Castracane VD. Endocrinology of preterm labor. Clin Obstet Gynecol. 2000; 43:717-26.

13. Smith R, Smith Jl, Shen X, Engel PJ, Bowman ME, McGrath SA, et al. Patterns of plasma corticotropin-releasing hormone, progesterone, estradiol, and estriol change and the onset of human labor. J Clin Endocrinol Metab. 2009;94:2066-74.

14. Glinoer $D$. The regulation of thyroid function in pregnancy: pathways of endocrine adaptation from physiology to pathology. Endocr Rev. 1997:18:404-33.

15. Smith R, Paul J, Maiti K, Tolosa J, Madsen G. Recent advances in understanding the endocrinology of human birth. Trends Endocrinol Metab. 2012:23:516-23.

16. Schug TT, Blawas AM, Gray K, Heindel JJ, Lawler CP. Elucidating the Links between Endocrine Disruptors and Neurodevelopment. Endocrinology. 2015:en20141734

17. Schug TT, Janesick A, Blumberg B, Heindel JJ. Endocrine disrupting chemicals and disease susceptibility. J Steroid Biochem Mol Biol. 2011;127:204-15.

18. Krause M, Klit A, Blomberg Jensen M, Søeborg T, Frederiksen H, Schlumpf M, et al. Sunscreens: are they beneficial for health? An overview of endocrine disrupting properties of UV-filters. Int J Androl. 2012;35:424-36.

19. Darbre PD, Harvey PW. Paraben esters: review of recent studies of endocrine toxicity, absorption, esterase and human exposure, and discussion of potential human health risks. J Appl Toxicol JAT. 2008;28:561-78.

20. Wang C-F, Tian Y. Reproductive endocrine-disrupting effects of triclosan: Population exposure, present evidence and potential mechanisms. Environ Pollut Barking Essex. 2015;206:195-201.

21. Giulivo M, Lopez de Alda M Capri E, Barceló D. Human exposure to endocrine disrupting compounds: their role in reproductive 
systems, metabolic syndrome and breast cancer. A review. Environ Res. 2016;151:251-64.

22. Di Renzo GC, Conry JA, Blake J, DeFrancesco MS, DeNicola N, Martin JN, et al. International Federation of Gynecology and Obstetrics opinion on reproductive health impacts of exposure to toxic environmental chemicals. Int J Gynecol Obstet. 2015;131:219-25.

23. Mallozzi M, Bordi G, Garo C, Caserta D. The effect of maternal exposure to endocrine disrupting chemicals on fetal and neonatal development: a review on the major concerns. Birth Defects Res Part C Embryo Today Rev. 2016:108:224-42.

24. Rattan S, Zhou C, Chiang C, Mahalingam S, Brehm E, Flaws J. Exposure to endocrine disruptors during adulthood: consequences for female fertility. J Endocrinol. 2017;233(3):R109-R129. https://doi.org/10.1530/JOE-17-0023

25. Braun JM. Early-life exposure to EDCs: role in childhood obesity and neurodevelopment. Nat Rev Endocrinol. 2017;13:161-73.

26. Aker AM, Watkins DJ, Johns LE, Ferguson KK, Soldin OP, Anzalota Del Toro LV, et al. Phenols and parabens in relation to reproductive and thyroid hormones in pregnant women. Environ Res. 2016;151:30-7.

27. Cantonwine DE, Cordero JF, Rivera-González LO, Anzalota Del Toro LV, Ferguson KK, Mukherjee B, et al. Urinary phthalate metabolite concentrations among pregnant women in northern Puerto Rico: distribution, temporal variability, and predictors. Environ Int. 2014;62:1-11.

28. Meeker JD, Cantonwine DE, Rivera-González LO, Ferguson KK, Mukherjee B, Calafat AM, et al. Distribution, variability, and predictors of urinary concentrations of phenols and parabens among pregnant women in Puerto Rico. Environ Sci Technol. 2013;47:3439-47.

29. Ye X, Kuklenyik Z, Needham LL, Calafat AM. Quantification of urinary conjugates of bisphenol a, 2,5-dichlorophenol, and 2-hydroxy-4methoxybenzophenone in humans by online solid phase extraction-high performance liquid chromatography-tandem mass spectrometry. Anal Bioanal Chem. 2005;383:638-44.

30. Ye X, Bishop AM, Reidy JA, Needham LL, Calafat AM. Parabens as urinary biomarkers of exposure in humans. Environ Health Perspect. 2006;1 14:1843-6.

31. Watkins DJ, Ferguson KK, Anzalota Del Toro LV, Alshawabkeh AN, Cordero $J F$, Meeker JD. Associations between urinary phenol and paraben concentrations and markers of oxidative stress and inflammation among pregnant women in Puerto Rico. Int J Hyg Environ Health. 2015;218:212-9.

32. Hornung RW, Reed LD. Estimation of average concentration in the presence of nondetectable values. Appl Occup Environ Hyg. 1990;5:46-51.

33. Dietrich JW, Landgrafe G, Fotiadou EH. TSH and Thyrotropic Agonists: Key Actors in Thyroid Homeostasis [Internet]. J. Thyroid Res. 2012;2012:29. Article ID 351864. https://doi.org/10.1155/2012/351864.

34. Romero R, Scoccia B, Mazor M, Wu YK, Benveniste R. Evidence for a local change in the progesterone/estrogen ratio in human parturition at term. Am J Obstet Gynecol. 1988;159:657-60.

35. Ruiz RJ, Saade GR, Brown CEL, Nelson-Becker C, Tan A, Bishop S, et al. The effect of acculturation on progesterone/estriol ratios and preterm birth in Hispanics. Obstet Gynecol. 2008;111:309-16.

36. O'Brien KM, Upson K, Cook NR, Weinberg CR. Environmental Chemicals in Urine and Blood: improving methods for creatinine and lipid adjustment. Environ Health Perspect. 2016;124:220-7.

37. Sartain CV, Hunt PA. An old culprit but a new story: bisphenol a and "NextGen" bisphenols. Fertil Steril. 2016;106:820-6.

38. Aker AM, Watkins DJ, Johns LE, Ferguson KK, Soldin OP, Del Toro LVA, et al. Phenols and parabens in relation to reproductive and thyroid hormones in pregnant women. Environ Res. 2016;151:30-7.

39. Aker AM, Johns L, McElrath TF, Cantonwine DE, Mukherjee B, Meeker JD. Associations between maternal phenol and paraben urinary biomarkers and maternal hormones during pregnancy: a repeated measures study. Environ Int. 2018;113:341-9.

40. Chevrier J, Gunier RB, Bradman A, Holland NT, Calafat AM, Eskenazi B, et al. Maternal urinary bisphenol a during pregnancy and maternal and neonatal thyroid function in the CHAMACOS study. Environ Health Perspect. 2013; 121:138-44.

41. Romano ME, Webster GM, Vuong AM, Thomas Zoeller R, Chen A, Hoofnagle AN, et al. Gestational urinary bisphenol a and maternal and newborn thyroid hormone concentrations: the HOME study. Environ Res. 2015;138: 453-60.

42. Aung MT, Johns LE, Ferguson KK, Mukherjee B, McElrath TF, Meeker JD. Thyroid hormone parameters during pregnancy in relation to urinary bisphenol a concentrations: a repeated measures study. Environ Int. 2017:104:33-40.

43. Park C, Choi W, Hwang M, Lee Y, Kim S, Yu S, et al. Associations between urinary phthalate metabolites and bisphenol a levels, and serum thyroid hormones among the Korean adult population - Korean National Environmental Health Survey (KoNEHS) 2012-2014. Sci Total Environ. 2017;584-585:950-7.

44. van de Beek C, Thijssen JHH, Cohen-Kettenis PT, van Goozen SHM, Buitelaar JK. Relationships between sex hormones assessed in amniotic fluid, and maternal and umbilical cord serum: what is the best source of information to investigate the effects of fetal hormonal exposure? Horm Behav. 2004;46:663-9.

45. Gonçalves GD, Semprebon SC, Biazi BI, Mantovani MS, Fernandes GSA. Bisphenol a reduces testosterone production in TM3 Leydig cells independently of its effects on cell death and mitochondrial membrane potential. Reprod Toxicol Elmsford N. 2017;76:26-34.

46. Mahalingam S, Ther L, Gao L, Wang W, Ziv-Gal A, Flaws JA. The effects of in utero bisphenol a exposure on ovarian follicle numbers and steroidogenesis in the F1 and F2 generations of mice. Reprod Toxicol Elmsford N. 2017;74:150-7.

47. Ferguson KK, Peterson KE, Lee JM, Mercado-García A, Blank-Goldenberg C, Téllez-Rojo MM, et al. Prenatal and peripubertal phthalates and bisphenol a in relation to sex hormones and puberty in boys. Reprod Toxicol Elmsford N. 2014:47:70-6.

48. Makieva S, Saunders PTK, Norman JE. Androgens in pregnancy: roles in parturition. Hum Reprod Update. 2014;20:542-59.

49. Aker AM, Ferguson KK, Rosario ZY, Mukherjee B, Alshawabkeh AN, Cordero $J$ F, et al. The associations between prenatal exposure to triclocarban, phenols and parabens with gestational age and birth weight in northern Puerto Rico. Environ Res. 2018;169:41-51.

50. Hines M, Golombok S, Rust J, Johnston KJ, Golding J. Team TALS of P and CS. Testosterone during pregnancy and gender role behavior of preschool children: a longitudinal, population study. Child Dev. 2002;73:1678-87.

51. Grino M, Chrousos GP, Margioris AN. The corticotropin releasing hormone gene is expressed in human placenta. Biochem Biophys Res Commun. 1987;148:1208-14.

52. Makrigiannakis A, Zoumakis E, Kalantaridou S, Coutifaris C, Margioris AN, Coukos $\mathrm{G}$, et al. Corticotropin-releasing hormone promotes blastocyst implantation and early maternal tolerance. Nat Immunol. 2001;2:1018-24.

53. Nezi M, Mastorakos G, Mouslech Z. Corticotropin releasing hormone and the immune/inflammatory response. In: De Groot L, Chrousos G, Dungan K, Feingold KR, Grossman A, Hershman JM, et al., editors. Endotext [internet]. South Dartmouth (MA): MDText.com, Inc:; 2000. Available from: http://www. ncbi.nlm.nih.gov/books/NBK279017/. Accessed 9 Mar 2018.

54. Kalantaridou SN, Zoumakis E, Makrigiannakis A, Godoy H, Chrousos GP. The role of corticotropin-releasing hormone in blastocyst implantation and early fetal immunotolerance. Horm Metab Res Horm Stoffwechselforschung Horm Metab. 2007:39:474-7.

55. McLean null, Smith null. Corticotropin-releasing Hormone in Human Pregnancy and Parturition. Trends Endocrinol Metab TEM. 1999;10:174-8.

56. Madhappan B, Kempuraj D, Christodoulou S, Tsapikidis S, Boucher W, Karagiannis $\mathrm{V}$, et al. High levels of intrauterine Corticotropin-releasing hormone, Urocortin, Tryptase, and Interleukin-8 in spontaneous abortions. Endocrinology. 2003;144:2285-90.

57. Arck PC, Rücke M, Rose M, Szekeres-Bartho J, Douglas AJ, Pritsch M, et al. Early risk factors for miscarriage: a prospective cohort study in pregnant women. Reprod BioMed Online. 2008;17:101-13.

58. Petraglia F, Imperatore A, Challis JRG. Neuroendocrine mechanisms in pregnancy and parturition. Endocr Rev. 2010;31:783-816.

59. Ni X, Hou Y, King BR, Tang X, Read MA, Smith R, et al. Estrogen receptormediated down-regulation of corticotropin-releasing hormone gene expression is dependent on a cyclic adenosine 3',5'-monophosphate regulatory element in human placental syncytiotrophoblast cells. J Clin Endocrinol Metab. 2004;89:2312-8.

60. Caserta D, Di Segni N, Mallozzi M, Giovanale V, Mantovani A, Marci R, et al. Bisphenol $\mathrm{a}$ and the female reproductive tract: an overview of recent laboratory evidence and epidemiological studies. Reprod Biol Endocrinol. 2014;12:37.

61. Tan W, Huang H, Wang Y, Wong TY, Wang CC, Leung LK. Bisphenol a differentially activates protein kinase $C$ isoforms in murine placental tissue. Toxicol Appl Pharmacol. 2013;269:163-8. 
62. Rajakumar C, Guan H, Langlois D, Cernea M, Yang K. Bisphenol a disrupts gene expression in human placental trophoblast cells. Reprod Toxicol Elmsford N. 2015;53:39-44.

63. Smith R, Nicholson RC. Corticotrophin releasing hormone and the timing of birth. Front Biosci J Virtual Libr. 2007;12:912-8.

64. Ruszkiewicz JA, Li S, Rodriguez MB, Aschner M. Is Triclosan a neurotoxic agent? J Toxicol Environ Health B Crit Rev. 2017;20:104-17.

65. Rodríguez PEA, Sanchez MS. Maternal exposure to triclosan impairs thyroid homeostasis and female pubertal development in Wistar rat offspring. J Toxicol Environ Health A. 2010;73:1678-88.

66. Wang $X$, Chen $X$, Feng $X$, Chang F, Chen M, Xia Y, et al. Triclosan causes spontaneous abortion accompanied by decline of estrogen sulfotransferase activity in humans and mice. Sci Rep. 2015;5:18252.

67. Cao X, Hua X, Wang $X$, Chen L. Exposure of pregnant mice to triclosan impairs placental development and nutrient transport. Sci Rep. 2017;7:44803.

68. Axelstad M, Boberg J, Vinggaard AM, Christiansen S, Hass U. Triclosan exposure reduces thyroxine levels in pregnant and lactating rat dams and in directly exposed offspring. Food Chem Toxicol Int J Publ Br Ind Biol Res Assoc. 2013;59:534-40.

69. Crofton KM, Paul KB, Devito MJ, Hedge JM. Short-term in vivo exposure to the water contaminant triclosan: evidence for disruption of thyroxine. Environ Toxicol Pharmacol. 2007;24:194-7.

70. Johnson PI, Koustas E, Vesterinen HM, Sutton P, Atchley DS, Kim AN, et al. Application of the navigation guide systematic review methodology to the evidence for developmental and reproductive toxicity of triclosan. Environ Int. 2016;92-93:716-28.

71. Louis GW, Hallinger DR, Braxton MJ, Kamel A, Stoker TE. Effects of chronic exposure to triclosan on reproductive and thyroid endpoints in the adult Wistar female rat. J Toxicol Environ Health A. 2017;80:236-49.

72. Paul KB, Thompson JT, Simmons SO, Vanden Heuvel JP, Crofton KM. Evidence for triclosan-induced activation of human and rodent xenobiotic nuclear receptors. Toxicol in Vitro. 2013;27:2049-60.

73. Paul KB, Hedge JM, Devito MJ, Crofton KM. Developmental triclosan exposure decreases maternal and neonatal thyroxine in rats. Environ Toxicol Chem. 2010;29:2840-4.

74. Paul KB, Hedge JM, DeVito MJ, Crofton KM. Short-term exposure to triclosan decreases thyroxine in vivo via upregulation of hepatic catabolism in young long-Evans rats. Toxicol Sci Off J Soc Toxicol. 2010;113:367-79.

75. Zorrilla LM, Gibson EK, Jeffay SC, Crofton KM, Setzer WR, Cooper RL, et al. The effects of triclosan on puberty and thyroid hormones in male Wistar rats. Toxicol Sci Off J Soc Toxicol. 2009;107:56-64.

76. Braun JM, Chen A, Hoofnagle A, Papandonatos GD, Jackson-Browne M, Hauser $\mathrm{R}$, et al. Associations of early life urinary triclosan concentrations with maternal, neonatal, and child thyroid hormone levels. Horm Behav. 2018;101:77-84.

77. Jackson-Browne MS, Papandonatos GD, Chen A, Calafat AM, Yolton K, Lanphear BP, et al. Identifying vulnerable periods of neurotoxicity to Triclosan exposure in children. Environ Health Perspect. 2018;126:057001.

78. Koeppe ES, Ferguson KK, Colacino JA, Meeker JD. Relationship between urinary triclosan and paraben concentrations and serum thyroid measures in NHANES 2007-2008. Sci Total Environ. 2013;445-446:299-305.

79. Feng Y, Zhang P, Zhang Z, Shi J, Jiao Z, Shao B. Endocrine disrupting effects of Triclosan on the placenta in pregnant rats. PLoS One. 2016; 11:e0154758.

80. Wang X, Ouyang F, Feng L, Wang X, Liu Z, Zhang J. Maternal urinary Triclosan concentration in relation to maternal and neonatal thyroid hormone levels: a prospective study. Environ Health Perspect. 2017;125:067017.

81. Witorsch RJ. Critical analysis of endocrine disruptive activity of triclosan and its relevance to human exposure through the use of personal care products. Crit Rev Toxicol. 2014;44:535-55.

82. Carlsen SM, Jacobsen G, Romundstad P. Maternal testosterone levels during pregnancy are associated with offspring size at birth. Eur J Endocrinol. 2006;155:365-70.

83. Vladeanu M, Giuffrida O, Bourne VJ. Prenatal sex hormone exposure and risk of Alzheimer disease: a pilot study using the 2D:4D digit length ratio. Cogn Behav Neurol Off J Soc Behav Cogn Neurol. 2014;27:102-6.

84. Ashrap P, Watkins DJ, Calafat AM, Ye X, Rosario Z, Brown P, et al. Elevated concentrations of urinary triclocarban, phenol and paraben among pregnant women in northern Puerto Rico: predictors and trends. Environ Int. 2018.
85. Watanabe Y, Kojima H, Takeuchi S, Uramaru N, Ohta S, Kitamura S. Comparative study on transcriptional activity of 17 parabens mediated by estrogen receptor $\alpha$ and $\beta$ and androgen receptor. Food Chem Toxicol Int J Publ Br Ind Biol Res Assoc. 2013;57:227-34.

86. Wróbel AM, Gregoraszczuk EŁ. Actions of methyl-, propyl- and butylparaben on estrogen receptor- $a$ and $-\beta$ and the progesterone receptor in MCF-7 cancer cells and non-cancerous MCF-10A cells. Toxicol Lett. 2014;230:375-81.

87. Vo TTB, Jung E-M, Choi K-C, Yu FH, Jeung E-B. Estrogen receptor a is involved in the induction of Calbindin-D(9k) and progesterone receptor by parabens in $\mathrm{GH} 3$ cells: a biomarker gene for screening xenoestrogens. Steroids. 2011;76:675-81.

88. Aker AM, Johns L, McElrath TF, Cantonwine DE, Mukherjee B, Meeker JD. Associations between maternal phenol and paraben urinary biomarkers and maternal hormones during pregnancy: A repeated measures study. Environ Int. 2018;113:341-9. https://doi.org/10.1016/j.envint.2018.01.006

89. Vo TTB, Yoo Y-M, Choi K-C, Jeung E-B. Potential estrogenic effect(s) of parabens at the prepubertal stage of a postnatal female rat model. Reprod Toxicol Elmsford N. 2010;29:306-16.

90. Janjua NR, Mortensen GK, Andersson A-M, Kongshoj B, Skakkebaek NE, Wulf HC. Systemic uptake of diethyl phthalate, dibutyl phthalate, and butyl paraben following whole-body topical application and reproductive and thyroid hormone levels in humans. Environ Sci Technol. 2007:41:5564-70.

91. Meeker JD, Yang T, Ye X, Calafat AM, Hauser R. Urinary concentrations of parabens and serum hormone levels, semen quality parameters, and sperm DNA damage. Environ Health Perspect. 2011;119:252-7.

92. Rousset B. Antithyroid effect of a food or drug preservative: 4hydroxybenzoic acid methyl ester. Experientia. 1981;37:177-8.

93. van den Boogaard E, Vissenberg R, Land JA, van Wely M, van der Post JAM, Goddijn $M$, et al. Significance of (sub)clinical thyroid dysfunction and thyroid autoimmunity before conception and in early pregnancy: a systematic review. Hum Reprod Update. 2011;17:605-19.

94. Tingi E, Syed AA, Kyriacou A, Mastorakos G, Kyriacou A. Benign thyroid disease in pregnancy: a state of the art review. J Clin Transl Endocrinol. 2016;6:37-49.

Ready to submit your research? Choose BMC and benefit from:

- fast, convenient online submission

- thorough peer review by experienced researchers in your field

- rapid publication on acceptance

- support for research data, including large and complex data types

- gold Open Access which fosters wider collaboration and increased citations

- maximum visibility for your research: over $100 \mathrm{M}$ website views per year

At BMC, research is always in progress.

Learn more biomedcentral.com/submissions 